\title{
Measurement and Interpretation of Productivity and Functional Correctness
}

\author{
Hakan Erdogmus
}

Measuring developer productivity and functional correctness is central to evaluating software practices and techniques. Researchers use a wide variety of measurement and reporting methods. As such, the interpretation, aggregation and comparison of experimental results become difficult. The problems often reduce to the proper ways of defining units of development work and quantifying developer output. For example, when is it appropriate to measure productivity in terms of elapsed problem solving time vs. output per unit time? Is number of lines of source code an eternally damned output measure in all situations? How do we define task completion to measure problem solving time? What are the consequences of having a cut-off time? When is a minimum quality or usability criterion necessary? How should such a criterion be defined? What are some good output metrics that proxy external functionality? How can we effectively measure these metrics? When is it acceptable to define functional correctness as a binary variable? What are the pros and cons of objective vs. subjective measures?

While defining measures that are universally meaningful and applicable is not feasible, researchers, reviewers, and end readers would benefit from metric selection and reporting guidelines for some common contexts. Selecting proper measures will remain largely a matter of reconciling the several trade-offs involved in experimental design. However, guidelines that explicitly identify these trade-offs may prevent their arbitrary resolution, thus both streamlining and facilitating their interpretation.

Emerging approaches for automated acceptance testing [1] and process telemetry [2] raise some interesting possibilities for the definition, measurement, and selection of quasi-standard measures of productivity and correctness. It would be worthwhile to investigate the possibility of leveraging these developments to assist experimental researchers.

\section{References}

1. H. Erdogmus, M. Morisio, M. Torchiano, " On the effectiveness of Test-Driven Development,” IEEE Trans. Software Engineering, 31(1), January 2005.

2. P.M. Johnson, et al, "Software Development Management through Software Project Telemetry," IEEE Software, 22(4), July 2005. 